Original Research Paper

\title{
Design and Simulation of Novel Gated Integrator for the Heavy ion Beam Monitors System
}

\author{
${ }^{1}$ Djamet Yimga Arnaud, ${ }^{2}$ Wembe Tafo Evariste and ${ }^{1}$ Essimbi Zobo Bernard \\ ${ }^{I}$ Department of Physics, Faculty of Science, University of Yaoundé I, P.O. Box 812 Yaoundé, Cameroon \\ ${ }^{2}$ Department of Physics, Faculty of Science, University of Douala, P.O. Box 24157 Douala, Cameroon
}

\author{
Article history \\ Received: 12-02-2017 \\ Revised: $17-02-2017$ \\ Accepted: 26-02-2017 \\ Corresponding Author: \\ Wembe Tafo Evariste \\ Department of Physics, Faculty \\ of Science, University of \\ Douala, P.O. Box 24157 \\ Douala, Cameroon \\ Email: twembee@yahoo.fr
}

\begin{abstract}
In this study, the objective is to realize a Gated Integrator (GI) circuit for silicon strip, $\mathrm{Si}(\mathrm{Li}), \mathrm{CdZnTe}$ and CsI detectors etc. With the development of radioactive ion beam physics, heavy-charged particles like carbon ions have been applied to the treatment of deep-seated inoperable tumors in the therapy terminal of the Heavy ion Research Facility in Lanzhou (HIRFL) located at the Institute of Modern Physics (IMP), Chinese Academy of Sciences (CAS). A high resolution current measurement circuit was developed to monitor the beam current at $1 \mathrm{pA}$ range. The circuit consisted of a low current high sensitivity I/V converter and gated integrator in an energy spectroscopy. A low offset voltage precision amplifier and new guarding and shielding techniques were used in the I/V converter circuit which allowed to measure low current. This paper will show a MOS switch configuration which is used to prevent leakage current and novel technique to compensate a charge injection in the reset switch.
\end{abstract}

Keywords: Energy Spectroscopy, Noise, Gated Integrator, Charge Injection, Leakage Current Prevention

\section{Introduction}

The basic concept of gated integrator is to integrate signal as well as background when the image appears, to block or subtract the background when there is no image signal. Such procedures are executed numerous times for summation and averaging. Widely used imaging systems such as Si Charge Coupled Device (CCD), Complementary Metal-Oxide field effect transistor Active Pixel Sensor (CMOS APS), Schottky-Barrier Detector (SBD), InGaAs, InSb and HgCdTe FPAs are all based on the concept of current integration to accumulate signal in the purpose of improving the Signal-to-Noise Ratio (SNR), dynamic range and sensitivity. In Cooling Storage Ring (CSR) IMP Lanzhou, the main works are heavy ion beam accumulation, experiments related to cancer therapy, patients' treatment, mass measurement and prophase experiments on recombination (Kong et al., 2010; Zhang and $\mathrm{Wu}, 2011$; Zhou et al., 2012). The main function of the electron cooler in the CSR is the accumulation of heavy ion beams. The efficiency of the accumulation was related with many parameters of storage ring and electron cooler, such as the work-point setting, closed-orbit, electron density and angle between the electron beam and the ion beam. Initially, the alignment of the electron beam was done to maximize the intensity of the accumulated ion beam. From November 2006, to 2012, around 106 patients have been irradiated in Lanzhou (HIRFL) at IMP, where carbon-ion beams with energies up to $100 \mathrm{MeV} / \mu$ can be supplied and a passive beam delivery system has been developed and commissioned (Li and Sihver, 2011). A number of therapeutic and clinical experiments (Kraft, 2000) on heavy-ion therapy were acquired at the IMP. To extend the heavy-ion therapy project to the treatment of a deep tumor, a horizontal beam line dedicated to this has been constructed in the CSR, which is a synchrotron connected to the HIRFL as an injector to be analyzed by detectors and is now in operation. A beam current monitoring circuit was necessary and urgent to calibrate these detectors. Beams applied to treatment are less than $1 \mathrm{pA}$. These low beams current are modulated because of operating modes of a synchrotron and also suffer from poor accuracy (Kong et al., 2010; Auzelyte et al., 2006).

This paper presents a description of a low current measurement circuit for the beam current monitor system. In detail the paper will describe the structure of GI based on leakage current, charge injection, noise 
analysis and introduction of a switch technique with high sensitivity when comes the measurement of low current. It also shows that the noise analysis during a transient voltage excursion appeared at the gate can sense the readout system.

\section{GI in the Beam Monitors System}

Figure 1 shows the block diagram of the heavy ion beam current monitor system. The low current measurement circuit consists of I/V Converter and a GI (Sun et al., 2003). The current signal from the Faraday cup (FC), which was pulsed for $0.5 \mathrm{~ms}$ a period of $4 \mathrm{~ms}$, was converted into a voltage signal through a $\mathrm{I} / \mathrm{V}$ Converter, the output of which was integrated by the GI in order to measure the charge of the beam (Kong et al., 2010; Redondo et al., 2007; Hori and Hanke, 2008). Outputs of I/V Converter and GI were acquired by a data acquisition system based on PXI.

Compared to traditional current gated integrators (Kong et al., 2010; Riboldi et al., 2010), which integrated the current from the FC by a GI directly, the new circuit prevented many disturbances like leakage current of the feedback capacitor and charge injection of switches.

\section{The Design of the New GI}

A gated integrator is designed to recover fast, repetitive, analog signals with time scales ranging from $10^{-11}$ to $10^{-4}$ seconds. In a typical application, a time window within a certain width is "gated" after a set delay from an internal or external trigger. A gated integrator amplifies and integrates the signal that is present during the time the gate is open, excluding noise and interference that may be present at other times. Since any signal present while the gate is open will add linearly, while noise will add in a "random walk" fashion as the square root of the number of shots, prevent leakage and charge injection compensation will improve the SNR. The gated integrator is actually a Capacitive
Transimpedance Amplifier (CTIA) except the gated switch, so it also performs as a low pass filter. To get the total charge of the beam, the output of the $\mathrm{I} / \mathrm{V}$ converter was fed into the GI, the schematic indicated by the dotted line in Fig. 1. The input signal voltage $V_{i}$ is converted into current via the resistor $R_{S}$ and integrated by the feedback capacitor $C_{f}$. The output voltage $V_{\text {out }}$ is given by:

$$
V_{\text {out }}=\frac{1}{C_{f} R_{S}} \int_{0}^{T} V_{i}(t) d t+V_{0}
$$

Since the input voltage $V_{i}$, is proportional to the current from the $F C$, the GI output $V_{\text {out }}$, represents the total charge of the beam for a given duration $T$. The leakage current elimination techniques and charge injection compensation were implemented in the GI section. The GI circuitry is composed of switches, operational amplifier and capacitors. The non-ideal switch and amplifier will introduce additional noise and fixed pattern (Levinzon, 2005; Wembe et al., 2014). The goal of the design is trying to understand the issues and take appropriate strategies to limit them such that the performance of the circuitry is not overshadowed.

\section{Characteristics of MOS Switch}

The main features of a MOS switch are switch speed, charge injection and clock feedthrough. The rise and fall times of a submicron gate switch is usually less than 0.1 ns. This can impose a problem only in readout circuit of extremely high speed Focal Plane Array (FPAs). The charge injection and clock feedthrough, however, are the main sources of noises in a MOS switch.

A switch is used to pass voltage or current. An ideal switch has the characteristics of zero resistance when it is $\mathrm{ON}$, infinite resistance when it is OFF and no delay when it is turned ON or OFF. A real MOSFET switch however, has turn-ON non-zero channel resistance, turn-OFF leakage current, threshold voltage and parasitic capacitances.

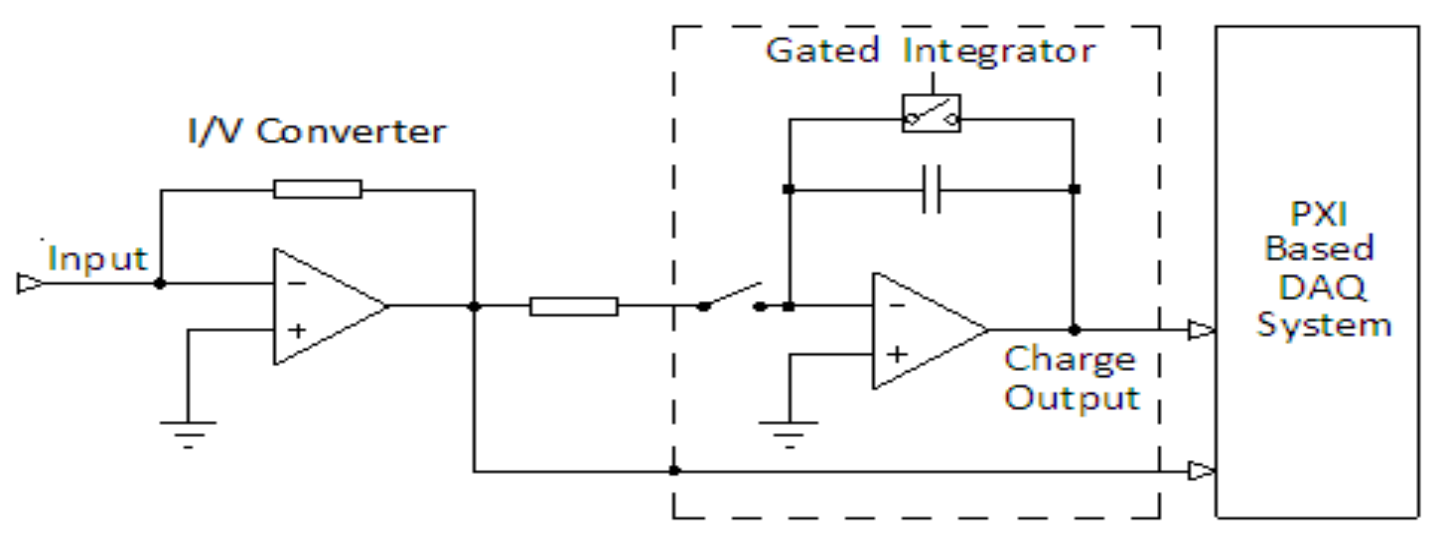

Fig. 1. Block diagram of the heavy-ion beam monitor system 


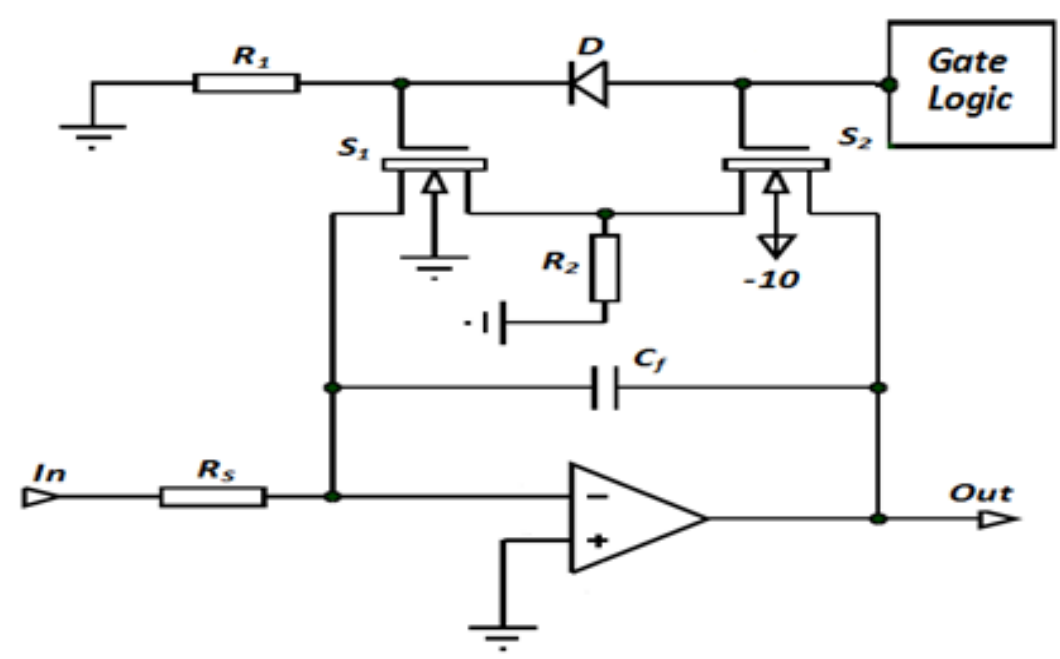

Fig. 2. A switch configuration as the reset switch

\section{MOS Switch Configuration to Prevent Leakage Current}

While the Gated Integrator (GI) is in "integration" or "hold" state, the effect reset switch has to be kept OFF. However the effect of leakage current in the GI reset switch will cause errors to the output in the long duration of these states. The problem can be resolved by using the CMOS switch configuration to replace single reset switch shown in Fig. 2.

Although both n-channel MOSFETs integrator, are switched together, $S_{1}$ is switched with gate voltages of zero and +5 volts so that gate leakage (as well as drainsource leakage) is entirely eliminated during the OFF state (zero gate voltage). In the $\mathrm{ON}$ state the capacitor is discharged as before, but with twice $R_{O N}$. In the OFF state, one has in $\mathrm{S}_{2}$ small leakage which passes to ground through $\mathrm{R}_{2}$ with negligible drop. There is no leakage current at the summing junction because $S_{1}$ 's source, drain and substrate are all at the same voltage. If the source and body are at the same potential, the drainsource current of a MOS transistor in the deep subthreshold region of operation is given by:

$$
\begin{array}{r}
I_{D}=K I_{D O} \cdot \exp \left(\frac{V_{G S}-V_{t h}}{\eta V_{T}}\right)\left(1-\exp \left(-\frac{V_{D S}}{V_{T}}\right)\right) \\
I_{D 0}=\mu_{n} C_{O X}(\eta-1) V_{T}^{2}
\end{array}
$$

where, $I_{D}$ is the drain to source current of the transistor MOS, $K$ is the aspect ratio of the transistor, $I_{D O}$ is the saturation current, $V_{G S}$ is the gate to source voltage, $V_{t h}$ is the threshold voltage of a MOSFET, $V_{T}$ is the thermal voltage, $V_{D S}$ is the drain to source voltage, $\mu_{n}$ is the carrier mobility, $C_{O X}$ is the gate oxide capacitance and $\eta$ is the sub-threshold slope factor (Redondo et al., 2007; Riboldi et al., 2010; Ueno et al., 2010 and Taur and Ning, 2002). Equation 2 shows, even for $V_{G S}=0$, the only way to establish a zero switch leakage current is to set $V_{D S}$ to $0 \mathrm{~V}$. To maintain the $V_{D S}$ of the MOSFET switch at virtual ground, a MOS switch configuration shown in Fig. 2, is used to replace the single reset switch from the gate integrator in Fig. 1. The MOS switch configuration is composed of two MOSFETs switches, $S_{1}, S_{2}$, in series and a grounded resistor, $R_{2}$, attached to the node between the two switches. When the two MOS switches in parallel with the storage capacitor are OFF, in $S_{2}$, a small leakage passes to ground through $R_{2}$ with negligible drop. There is no leakage current at the summing junction because $S_{1}$ 's source, drain and substrate are all at the same voltage. Thus, in this configuration, $V_{D S}$ of the switch connected to the inverting input of the integrator is maintained at $O V$ and zero or very little leakage current flows through this switch.

\section{Charge Injection Compensation}

MOS switches were chosen as switches in the GI. When a transient voltage excursion appeared at the gate, there would be an injection of electric charge into analog path via the gate-to-drain and the gate-to-source capacitances, which would cause errors to the GI output. The circuitry for electrical injection indicated by the dotted line in Fig. 3 was used to compensate error cause by this charge injection. When the state of switches is changed, certain quantity of charge will be injected into the inverting input via the capacitor $C_{\mathrm{C}}$ by the compensation network. This is opposite to the charge injected by switches. Since it is known that the amount of charge injected is equal to the product of the voltage excursion amplitude on the component and its 
capacitance, generating a product equal magnitude but opposite polarity can be used to compensate for this effect. After analysis and experimental trials, $C_{\mathrm{C}}$ was chosen to be $10 \mathrm{pF}$ from the Equation 3. Thus by changing the amplitude of the voltage excursion on $C_{\mathrm{C}}$, the error of the output voltage caused by charge injection was reduced to less than $0.7 \mathrm{mV}$ :

$$
\begin{aligned}
& Q^{f}=C_{C} V_{S}^{f}+C_{G S}\left(V_{S}^{f}-V_{G}\right) \text { when } \\
& V_{S}^{f}=\frac{R_{1}^{\prime} V_{E E}+R_{2}^{\prime} V_{G}}{R_{1}^{\prime}+R_{2}^{\prime}}
\end{aligned}
$$

where, $Q^{f}$ which is the final charges stored at node $V_{S}, V_{S}^{f}$ is the final stable voltage at node $V_{S}, V_{G}$ is the gate logic voltage, $V_{E E}$ is biasing voltage for the injection compensation circuit, $C_{G S}$ is the gate-to-source capacitance, $R_{1}^{\prime}$ and $R_{2}^{\prime}$ are resistances of the potentiometer and $C_{\mathrm{C}}$ the capacitor of the compensation network.

\section{Measurements and Discussions}

The measurements were developed in the laboratory in order to characterize DC and AC performances and efficiency of the circuit. The streaming signal generator XLV1 associated with voltage controlled current source was selected to supply input current signal to GI on the software program PSPICE. Specification of the New GI is shown in Table 1. Parameters were measured when the source current was in DC mode. All DC test results presented here, including linearity shown in Fig. 4.

In order to recover the signal from the measurement system, simulation from Fig. 5, shows how single switch does not prevent leakage current. Then it will be difficult to recover the total signal. To prevent this effect, the single reset switch is replaced by the MOS switch configuration shown in Fig. 2, then the leakage current will disappear see Fig. 6, but we still remark an error appearance when the switch turns ON. This error is from the charge injection. During the rising edge of the clock signal of the gate, the gate starts at 0 volts and increases toward high voltage. In the transition from 0 to $V_{i n}+V_{t h}$, the switch is OFF. Consequently, this part of the clock waveform can couple to $C_{i n t}$ via $C_{G S}$. As a result, a portion of the clock signal appears across $C_{i n t}$ as indicated on the Fig. 6, during the transition at 3.8 ms. The circuitry indicated by the dotted line in Fig. 3, was used to compensate the charge injection see the simulation result in Fig. 7.

In contrast to charge injection when the switch is turned from ON to OFF, there is charge absorption when the switch is turned from OFF to ON for successive readout signal, which will cause an error shown in Fig. 8. for second and third readout during a low current measurement. We solve the problem by using a slow change clock to turn OFF the switch, Fig. 9. This results because the charges in the channel will be mostly injected into the substrate.

Table 1. Specifications of the new GI

\begin{tabular}{ll}
\hline Parameter & Value \\
\hline Full scale output & $\pm 5 \mathrm{~V}$ \\
Voltage conversion gain & $-1.0 \mathrm{~V} / \mathrm{V}$ \\
Linearity error & $<0.06 \%$ \\
Output voltage noise & $<0.7 \mathrm{mV}(\mathrm{rms})$ \\
Output offset voltage & $<0.5 \mathrm{mV}(\mathrm{rms})$ \\
Injected charge & $<116 \mathrm{pC}$ \\
Temperature range & $-40^{\circ} \mathrm{C}$ to $125^{\circ} \mathrm{C}$ \\
\hline
\end{tabular}

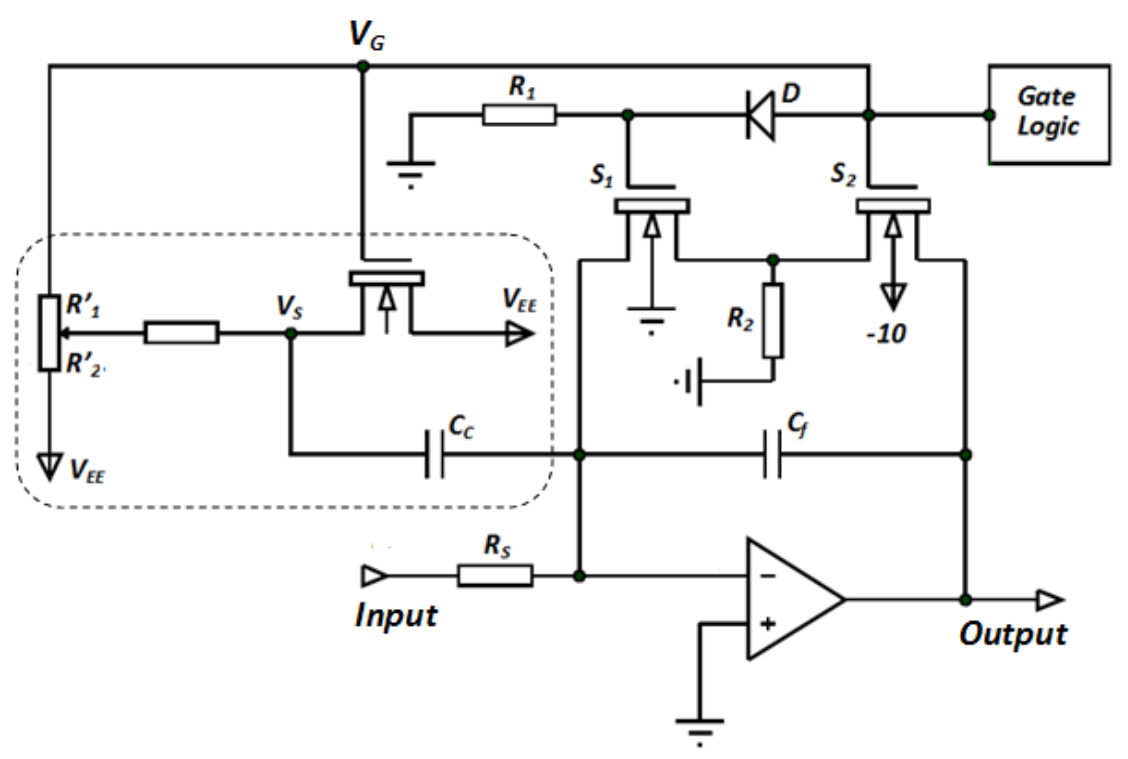

Fig. 3. Schematics of the GI with a network for charge injection compensation 


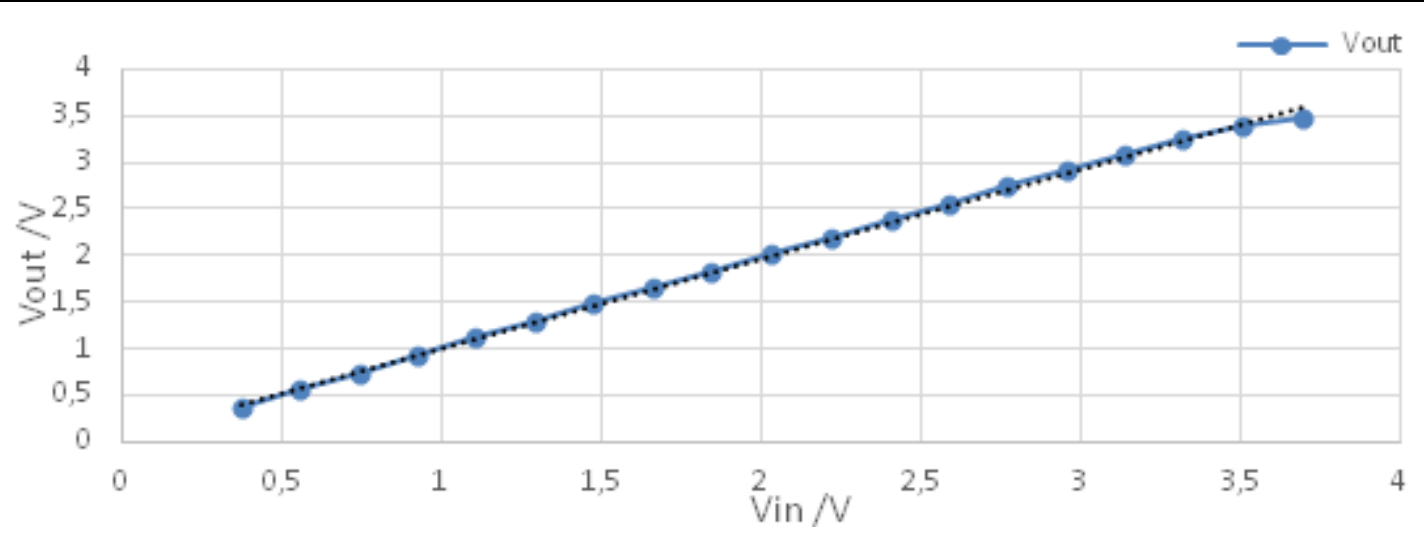

Fig. 4. Linearity of the GI

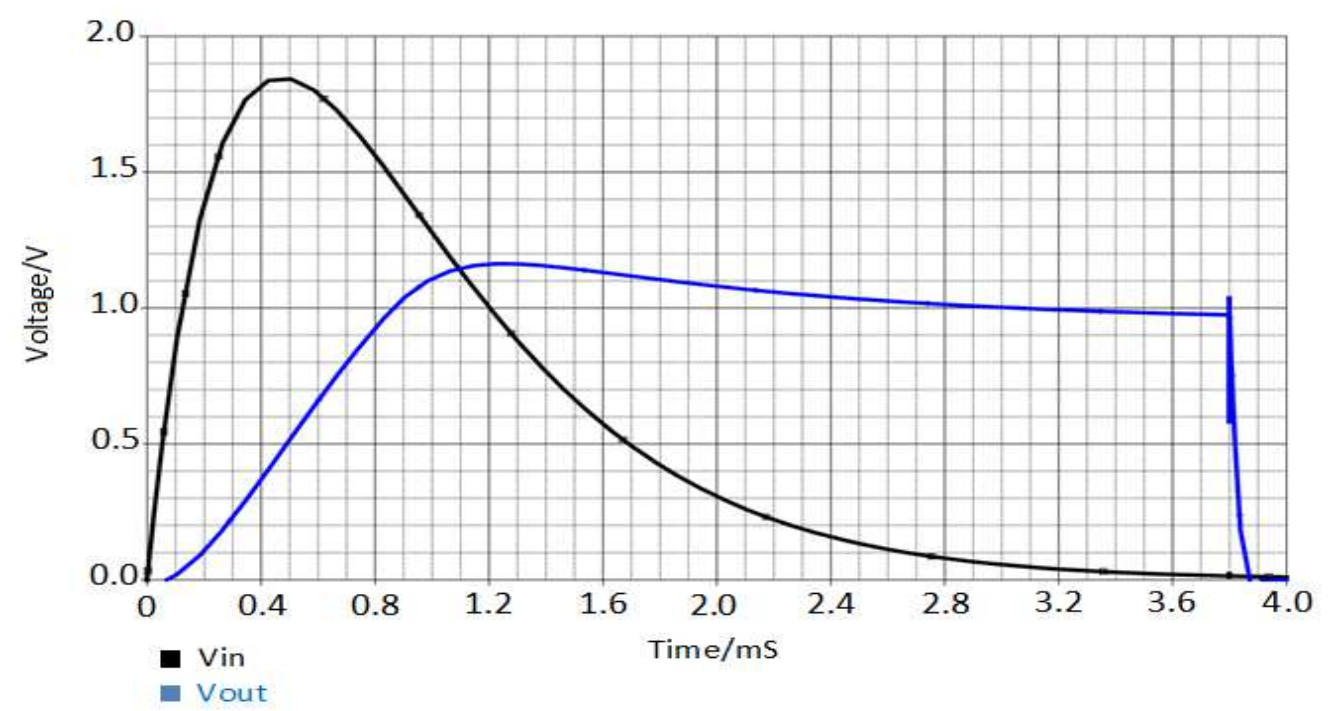

Fig. 5. Output waveform of the GI from I/V Converter output using a single reset switch

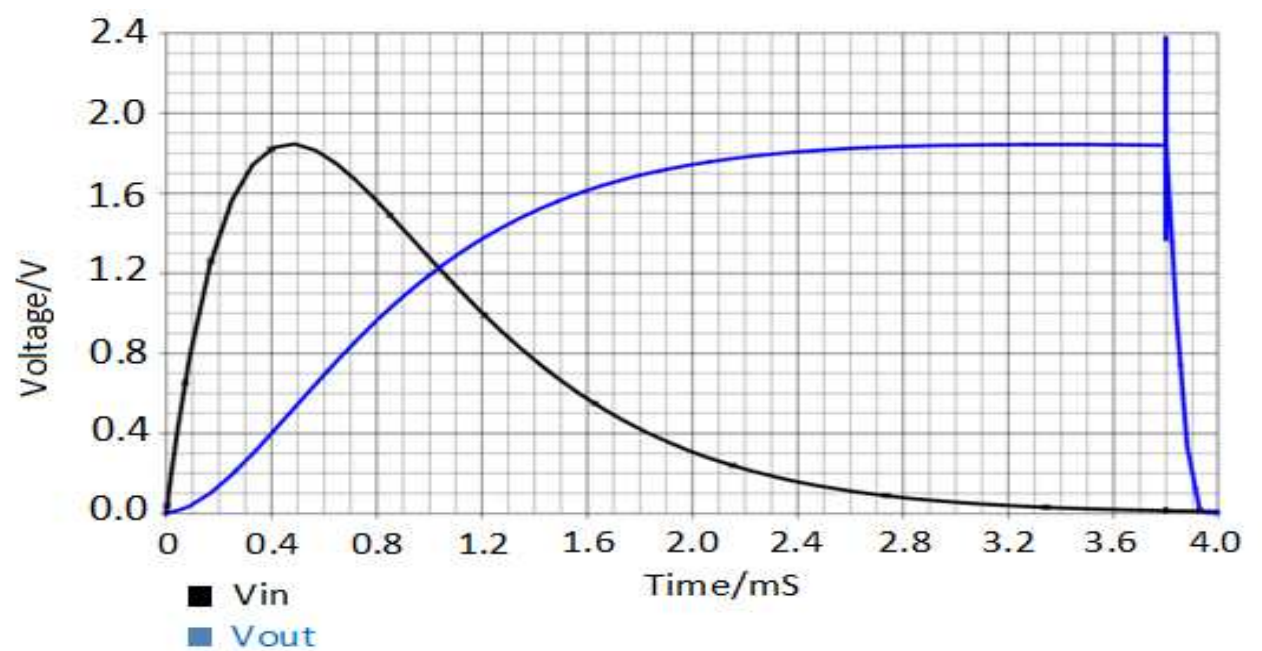

Fig. 6. Output waveform of the GI from I/V Converter output using switch configuration made by two NMOSFETs 


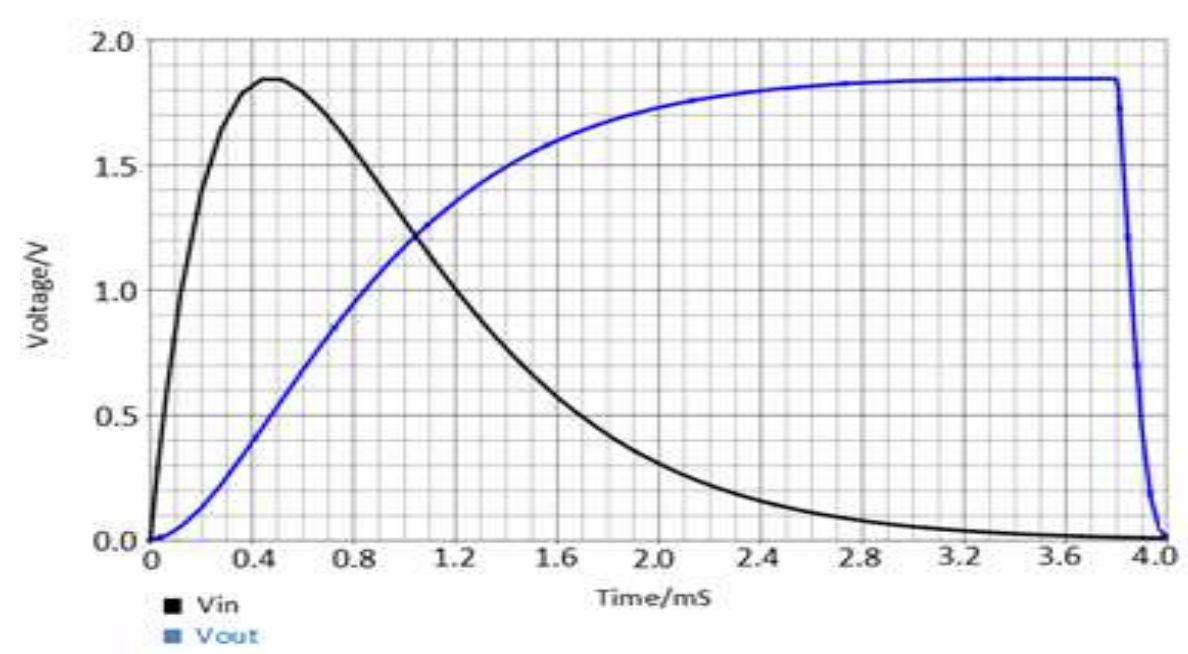

Fig. 7. Output waveform of the GI from I/V Converter output using switch configuration made by two transmissions gated to prevent charge injection

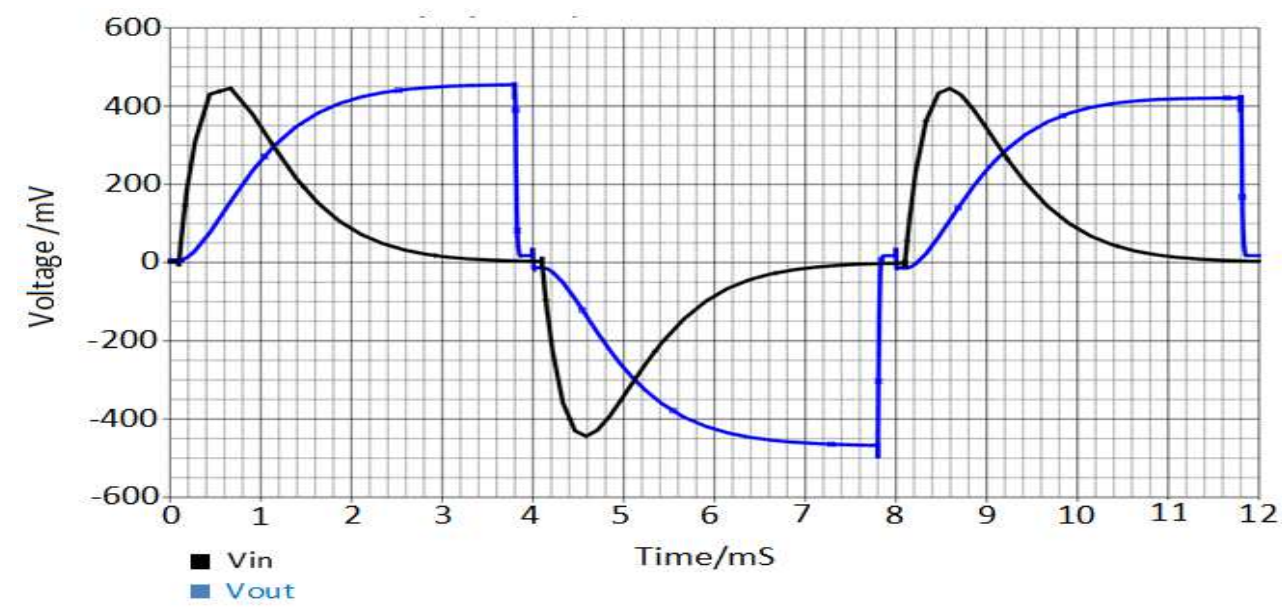

Fig. 8. Fast change clock to turn OFF the switch

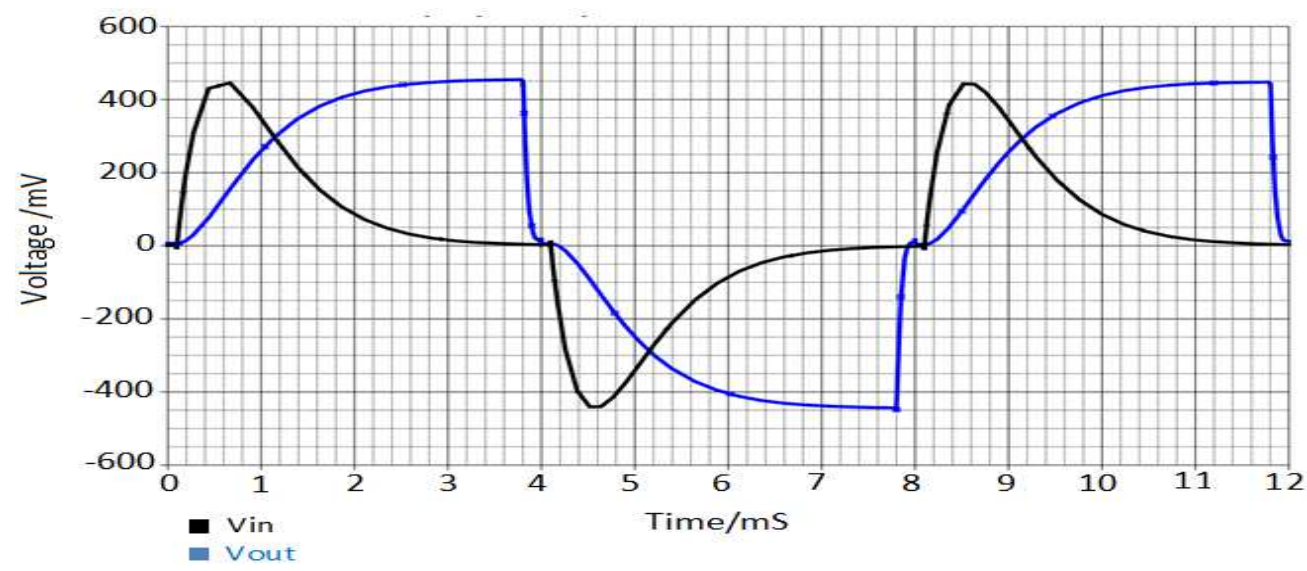

Fig. 9. Slow change clock to turn OFF the switch 


\section{Noise $(\mathrm{mV})$}

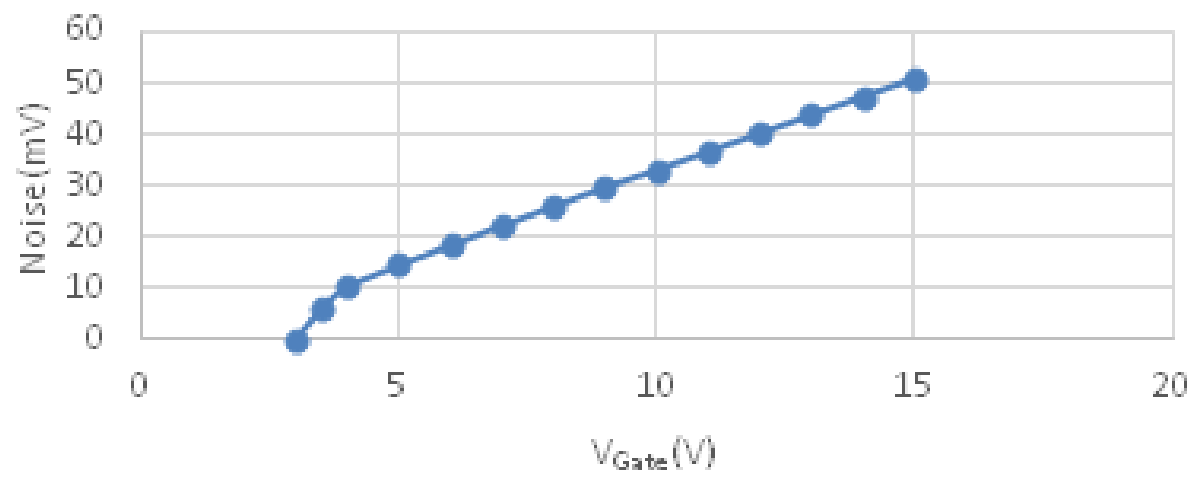

Fig. 10. Noise effect for the integrator versus clock voltage gate

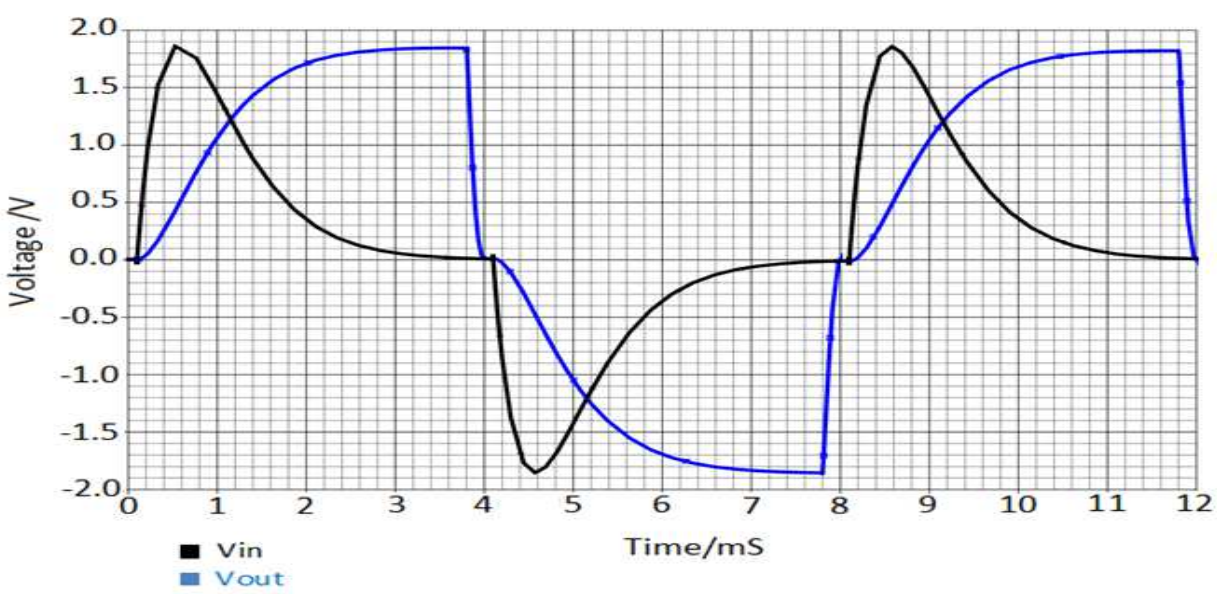

Fig. 11. Fast and slow change clock to turn OFF the switch with substantial value measurement

The injected charge induces fixed pattern noise, which will limit the minimum detectable signal. To choose a proper voltage for the clock signal of the gate to reduce pattern noise even though we use a rapid change clock, the analysis of noise effect is developed. The simulation results show that the number of these charges varies with the voltage of the gate. They are shown in the Fig. 10. One can just find a difference among the Fig. 8, 9 and 11. The simulation results show how noise effect appears when we are measuring low values and disappear with slow change clock. This noise effect is also negligible or disappears when the measurement is substantial.

\section{Conclusion}

After designing the new circuit, computer simulation was carried out by PSPICE simulator. The high degree of linearity was achieved by using a novel switch configuration, a new compensation approach which reduced the charge injection from switches in the GI to 0.7 $\mathrm{mV}$. The results of the simulation show how noise effect appears when we are measuring low values and disappear with slow change clock. This noise effect is also negligible or disappears when the measurement is substantial. The results of the simulation of a low and medium current measurement circuit show that it had a high resolution and stability. Because of the circuit design techniques used for high event rate capability insured that the circuit would perform well in high-resolution energy systems for the heavy ion beam monitors.

\section{Acknowledgement}

This work was supported in part by National Program on Key Basic Research Project of China (973 Program) (No. 2010CB834204), in part by the National Natural Science Foundation of China (No. 10735060), in part by the Third World Academy of Sciences and in part by the Important Direction Project of the CAS Knowledge Innovation Program (No. KJCX2-YW-N27). We thank the Director of Electronic Laboratory, Pr SU Hong and the staff for the logistic and hospitality. 


\section{Author's Contributions}

Wembe Tafo Evariste: Project leader, data interpretation and contribute to the writing of the paper.

Djamet Yimga Arnaud: Design and simulations model. Also, contribute to the writing of the paper.

Essimbi Zobo Bernard: Project leader. Revise and improve the final drafts of the paper.

\section{Ethics}

The corresponding author confirms that all of the other authors have read and approved the manuscript and no ethical issues involved.

\section{References}

Auzelyte, V., F. Andersson, M. Elfman, P. Kristiansson and J. Pallon et al., 2006. On-line measurement of proton beam current in pA range. Nucl. Instrum. Meth. Phys. Res. B, 249: 760-763.

DOI: 10.1016/j.nimb.2006.03.134

Hori, M. and K. Hanke, 2008. Spatial and temporal beam profile monitor with nanosecond resolution for CERN's Linac4 and Superconducting Proton Linac. Nucl. Instrum. Meth. Phys. Res. A, 588: 359-374. DOI: 10.1016/j.nima.2008.01.078

Kong, J., S. Hong, Z.Q. Chen, C.F. Dong and Y. Qian et al., 2010. Development of multi-channel gated integrator and PXI-DAQ system for nuclear detector arrays. Nucl. Instrum. Meth. Phys. Res. A, 622: 215-218. DOI: 10.1016/j.nima.2010.07.030

Kraft, G., 2000. Tumor therapy with ion beams. Nucl. Instrum. Meth. A, 454: 1-10

Levinzon, F.A., 2005. Noise of piezoelectric accelerometer with integral FET amplifier. IEEE Sensors J., 5: 1235-1242.

DOI: 10.1109/JSEN.2005.859256.

Li, Q. and L. Sihver, 2011. Therapeutic techniques applied in the heavy-ion therapy at IMP. Nucl. Instrum. Meth. Phys. Res. Sect. B, 269: 664-670. DOI: 10.1016/j.nimb.2011.01.125
Redondo, L.M., J. Rocha and J.C. Soares, 2007. A lowcost, accurate and non-intercepting continuous method for beam current measurements in a highcurrent ion implanter. Nucl. Instrum. Meth. Phys. Res. B, 265: 576-580. DOI: $10.1016 /$ j.nimb.2007.09.042

Riboldi, S., C. Cattadori, A. D'Andragora, A. Pullia and F. Zocca et al., 2010. A low-noise charge sensitive preamplifier for ge spectroscopy operating at cryogenic temperature in the GERDA experiment. Proceedings of the IEEE Nuclear Science Symposium Conference Record, Oct. 30-Nov. 6, IEEE Xplore Press, pp: 1386-1388. DOI: $10.1109 /$ NSSMIC.2010.5873998

Sun, Z., W.L. Zhan, Z.Y. Guo, G. Xiao and J.X. Li, 2003. RIBLL, the radioactive ion beam line in Lanzhou. Nucl. Instrum. Meth. A, 503: 496-503. DOI: $10.1016 / \mathrm{S} 0168-9002(03) 01005-2$

Taur, Y. and T.H. Ning, 2002. Fundamentals of modern VLSI devices. Cambridge Univ. Press, Cambridge, U.K.

Ueno, K., T. Hirose, T. Asai and Y. Amemiya, 2010. A $1-\mu \mathrm{W}$ 600-ppm/ ${ }^{\circ} \mathrm{C}$ current reference circuit consisting of subthreshold cmos circuits. IEEE Trans. Circuits Syst., 57: 681-685. DOI: 10.1109/TCSII.2010.2056051

Wembe, T.E., M.I. Adolphe, T. Daniel and D.Y. Arnaud, 2014. Noise optimization of readout front ends in CMOS technology with PS circuit. Asian J. Applied Sci., 2: 752-761.

Zhang, Y. and J. Wu, 2011. An improved forward travelling wave structure design for stochastic cooling at experimental cooler storage ring (CSRE). Proceedings of the on Beam Cooling, (BC' 11), pp: 132-135.

Zhou, C., S. Hong, R.S. Mao, C.F. Dong and Y. Qian et al., 2012. An accurate low current measurement circuit for heavy iron beam current monitor. Nucl. Instrum. Meth. Phys. Res. B, 280: 84-87.

DOI: 10.1016/j.nimb.2012.01.033 